\title{
Traditional Chinese Medicine Lingguizhugan Decoction Ameliorate HFD-Induced Hepatic-Lipid Deposition in Mice by Inhibiting STING-Mediated Inflammation in Macrophages
}

\section{Lin Cao ( $\nabla$ guodongcl@163.com )}

Nanjing University of Chinese Medicine

\section{Erjin Xu}

Nanjing University of Traditional Chinese Medicine: Nanjing University of Chinese Medicine

\section{Rendong Zheng}

Nanjing University of Traditional Chinese Medicine: Nanjing University of Chinese Medicine

\section{Rongling Zhong}

Jiangsu Provincial Institute of Traditional Chinese Medicine: Jiangsu Province Academy of Traditional Chinese Medicine

\section{Fei Huang}

Nanjing University of Traditional Chinese Medicine: Nanjing University of Chinese Medicine Juan Ye

Jiangsu Province Academy of Traditional Chinese Medicine

\section{Hongping Sun}

Nanjing University of Traditional Chinese Medicine: Nanjing University of Chinese Medicine

\section{Yaofu Fan}

Jiangsu Provincial Institute of Traditional Chinese Medicine: Jiangsu Province Academy of Traditional Chinese Medicine

\section{Shaofeng Xie}

Jiangsu Provincial Institute of Traditional Chinese Medicine: Jiangsu Province Academy of Traditional Chinese Medicine

\section{Yu Chen}

Jiangsu Provincial Institute of Traditional Chinese Medicine: Jiangsu Province Academy of Traditional Chinese Medicine

\section{Yijiao Xu}

Jiangsu Provincial Institute of Traditional Chinese Medicine: Jiangsu Province Academy of Traditional Chinese Medicine

\section{Zhili Zhangchen}

Nanjing University of Traditional Chinese Medicine: Nanjing University of Chinese Medicine Jing Cao 
Nanjing University of Traditional Chinese Medicine: Nanjing University of Chinese Medicine

Wen Cao

nanjingshi jiangning yiyuan: The Affiliated Jiangning Hospital of Nanjing Medical University

Chao Liu

Jiangsu Provincial Institute of Traditional Chinese Medicine: Jiangsu Province Academy of Traditional Chinese Medicine

\section{Research}

Keywords: Lingguizhugan decoction (LGZG)区hepatic steatosis, STING, Inflammation

Posted Date: May 27th, 2021

DOI: https://doi.org/10.21203/rs.3.rs-535051/v1

License: (c) (i) This work is licensed under a Creative Commons Attribution 4.0 International License. Read Full License

Version of Record: A version of this preprint was published at Chinese Medicine on January 5th, 2022. See the published version at https://doi.org/10.1186/s13020-021-00559-3. 


\section{Abstract}

Background: Stimulator of IFN genes (STING) is highly expressed in the livers of non-alcoholic fatty liver disease(NAFLD) patients and HFD induced NAFLD mice model. The STING signaling induced inflammation has been shown to be a critical role in metabolic disorders, such as NAFLD and Type 2 diabetes. Lingguizhugan decoction (LGZG), a Traditional Chinese herbal decoction, has been applied to treat metabolic disorders for many years. However, whether LGZG can alleviate the progression of NAFLD through inhibiting inflammation is not fully understood. This study was to determine the role of STING-mediated inflammation in the HFD- induced hepatic-lipid deposition treated with LGZG.

Methods: The anti-inflammatory and anti- hepatic-lipid deposition effects of LGZG in vivo were detected by H\&E staining, immunofluorescence and immunochemistry. Mice bone-marrow-derived macrophages (BMDMs) were co-incubated with palmitic acid-induced lipid deposition HepG2 cell model (BMDM+PAHepG2) and treated with LGZG.STING-specific agonist or blockers respectively to detect whether the activation of STING-mediated pathway is involved in the anti-hepatocyte lipid deposition effect of LGZG. Mitochondrial DNA was detected by real time PCR. The expression of inflammatory cytokines related to STING-TBK1-NF-KB pathway was detected by western blotting and ELISA.

Results: LGZG significantly ameliorated HFD induced hepatic steatosis, alleviated insulin resistance (IR) and reduced the oxidative stress. Furthermore, LGZG reduced hepatic mitochondrial damage and mitochondrial DNA release, which corresponded to reducing the expression of STING as well as the the infiltration of STING-positive Kupffer cells in the liver of HFD fed mice. LGZG directly inhibited the activation of STING-TBK1-NF-KB pathway in BMDM induced by DMXAA, LPS, thereby reducing the release of IFN $\beta$ and TNFa. Co-incubation LGZG treated BMDM and PA-stimulated HepG2 significantly reduced PA-induced lipid deposition in HepG2 cells by inhibiting STING- mediated signal pathways.

Conclusion: This study demonstrates that LGZG can ameliorate HFD-induced hepatic -lipid deposition through inhibiting STING-TBK1-NF-KB pathway in macrophages, which provides novel insight for elucidating the molecular mechanism of LGZG alleviating HFD induced hepatic steatosis.

\section{Background}

Non-alcoholic fatty liver disease (NAFLD) refers to liver injury caused by metabolic stress closely related to insulin resistance (IR) and genetic susceptibility. The disease spectrum includes non-alcoholic hepatic steatosis, nonalcoholic steatohepatitis (NASH), liver cirrhosis and hepatocellular carcinoma (HCC)[1]. In recent years, the incidence of NAFLD has risen sharply. The prevalence rate in the general population is $10-16 \%$, and the detection rate in obese patients is as high as $38 \%[2]$. NAFLD is a clinicopathological syndrome dominated by hepatocyte steatosis caused by genetic-environmental-metabolic stress related factors, and its pathogenesis has not been fully elucidated. It is currently believed to be closely related to obesity, aging, abnormal intestinal flora, diabetes, especially insulin resistance(IR). 
Recently, a large number of studies have shown that inflammation plays a key role in the process of NAFLD [3]. Inflammatory factors and inflammatory response run through all stages of NAFLD progression $[4,5]$. Alleviating liver inflammation can significantly slow down the progression of NAFLD [6-8]. Transmembrane protein 173 (TMEM173), also known as Stimulator of IFN genes (STING), is highly expressed in macrophages and becomes one of the core molecules involved in macrophages' resistance to virus invasion[9]. The STING signaling has been shown to be a critical role in metabolic disorders, antitumor immunity, infectious and inflammatory diseases through the recognition of bacterial DNA or self DNA[10]. When abnormal double-stranded DNA (such as bacterial, viral DNA, or DNA released by damaged mitochondria, mtDNA) is swallowed by macrophages into the cytoplasm, cyclic guanosine phosphate and adenosine phosphate are activated to form cyclic guanosine phosphate-adenosine phosphate (CGAMP) [11]. STING on the endoplasmic reticulum will be recognized and binded to cGAMP and then activated[11]. The activation of STING pathway simultaneously triggers TANK-binding kinase 1 (TBK1), which induces the phosphorylation of both interferon regulatory factor 3 (IRF3) [11] and NF-KB pathway[12], subsequently increasing the expression of type I interferon (IFN) and TNF-a[11]. STINGmediated signaling pathways play a key role in both liver macrophage-induced inflammation and IRinduced glucose as well as lipid metabolism disorders $[13,14]$.

Traditional Chinese medicine(TCM) has been widely used in the treatment of metabolic diseases for years with good efficacy and safety. Lingguizhugan decoction (LGZG) is an ancient Chinese herbal formula from a classic book of TCM titled Shanghan Zabing Lun. LGZG has been applied to treat metabolic diseases for many years and exhibited effects in alleviating obesity, hyperglycemia, hyperlipidemia, hypertension and hepatic injury[15-18]. Previous clinical studies have shown that LGZG can significantly alleviate liver steatosis, reduce TG, ALT and other biochemical indicators in NAFLD patients [15-18]. More importantly, studies have shown that LGZG elicit significantly anti-inflammatory effects[19]. However, due to the complex composition of TCM formulas that may involve multiple targets, whether LGZG can reduce lipid deposition in NAFLD by relieving inflammation is not fully understood. Here we proved that LGZG could reduce the release of inflammatory factors via inhibiting STINGmediated signaling pathways in liver macrophages, thereby ameliorating HFD-induced hepatic-lipid deposition in mice. This study will provide new insights for the expounding of the molecular mechanism of LGZG treatment in alleviating NAFLD.

\section{Materials And Methods}

\section{Reagents}

The four crude herbs, Poria cocos (PC, FuLing, Cat. No.20200102-1), Ramulus Cinnamomi (RC, GuiZhi, Cat. No.20200201-1), Rhizoma Atractylodis Macrocephalae (RAM, BaiZhu, Cat. No.2011010) and Radix Glycyrrhizae (RG, GanCao, Cat. No.20191001) were purchased from the Affiliated Hospital of Integrated Traditional Chinese and Western Medicine and authenticated by Professor Fangshi Zhu. All the crude drugs were morphologically authenticated according to Chinese Pharmacopoeia (2020 Edition).

Reference substances including cinnamaldehyde, Atractylenolide II and glycyrrhizic acid were purchased 
from PUSH Bio-Technology Co. Ltd (Chengdu, China). Lipopolysaccharide(LPS), STING specific inhibitor C176 and STING specific agonist DMXAA were purchased from MedChemExpress (MCE, NJ, USA). High fat diet(HFD) was purchased from New Brunswick(Cat. No. D12492, NJ, USA). Antibodies against STING, F4/80, TBK1, Phosphorylated TBK1(p-TBK1), TNFa, 8-OHdG, 4-hydroxy-2,3-E-nonenal (4-HNE) and 3Nitrotyrosine (3-NT) were purchased from CST(CST, GER). Enzyme-linked immunosorbent assay (ELISA) kits were purchased from R\&D (R\&D Systems, US). DNA extraction kit, First Strand cDNA Synthesis Kit and $2 \times$ SYBR Green/ROX qPCR Mix kit were purchased from Vazyme Biotech (Nanjing, Jiangsu, China). TRIzol reagent was purchased from Invitrogen (Carlsbad, CA, USA). Microplate assay kits for Blood gamma glutamyl transferase (GGT), alanine aminotransferase (ALT), aspartate aminotransferase (AST), triglyceride (TG) and total cholesterol (TC) measurement were purchased from Beyotime Biotechnology(Beyotime, Beijing, China).

\section{Preparation of LGZG}

LGZG, consisting of PC, RC, RAM and RG in the ratio of 4:3:3:2, were boiled twice with 10 times of deionized water $\left(\mathrm{ddH}_{2} \mathrm{O}, \mathrm{w} / \mathrm{v}\right)$ for one hour per time. The raw drug extracts was concentrated and lyophilized. The lyophilized powder was weighed and dissolved in $\mathrm{dd}_{2} \mathrm{O}$ for further use. The HPLC analysis was performed to identify the main compounds in LGZG $(5 \mathrm{mg} / \mathrm{mL})$ as previously reported [20]. Briefly, HPLC analysis was performed to identify the main compounds in LGZG. Chromatographic

separation was performed using an Waters XBridge C18 column ( $4.6 \mathrm{~mm} \times 250 \mathrm{~mm}, 5 \mu \mathrm{m}$; Waters,USA) at $30{ }^{\circ} \mathrm{C}$. The mobile phase consisted of water containing $0.1 \%$ Formic acid $(A)$ and Methanol $(B)$. The gradient program was set as follows: $0-5 \mathrm{~min}, 5-12 \% \mathrm{~B} ; 5-20 \mathrm{~min}, 12-14 \% \mathrm{~B} ; 20-40 \mathrm{~min}, 14-30 \% \mathrm{~B}$; 40-50 min, 30-35\% B; 50-60 min, 35-43\% B; 60-70 min, 43-50\% B; 70-75 min, 50-75\% B; 75-90 min, $75-85 \%$ B; $90-95 \mathrm{~min}, 85-95 \%$ B; $95-95.1 \mathrm{~min}, 95-5 \%$ B. The mobile phase flow rate was $1 \mathrm{~mL} / \mathrm{min}$, and the sample injection volume was $100 \mu \mathrm{L}$.

\section{Animals, feeding, and treatment}

6 to 8-week-old male C57BL/6J mice $18 \sim 20 \mathrm{~g} \rrbracket$ were feed in Experimental Animal Center, Affiliated Hospital of Integrated Traditional Chinese and Western Medicine (Nanjing, China). Mice were grouphoused in a pathogen-free animal facility under a $24 \mathrm{~h} \mathrm{light/dark} \mathrm{cycle} \mathrm{with} \mathrm{free} \mathrm{access} \mathrm{to} \mathrm{water} \mathrm{and}$ food. The experiments program were reviewed and approved by the Animal Ethics Committee of Affiliated Hospital of Integrated Traditional Chinese and Western Medicine with reference to European Community guidelines for the use of experimental animals. The mice were fed with standard chow diet $(10 \% \mathrm{kcal}$ from fat, set as the normal control group, $n=9$ ) or a high-fat diet (HFD) composed of $60 \%$ fat and $20 \%$ carbohydrate $(n=36)$. After 8 weeks feeding, the HFD fed mice were randomly divided into four groups $(\mathrm{n}=9)$ including Model (Mod), LGZG-L (low dose of LGZG, at a dose of $11 \mathrm{~g} / \mathrm{kg} /$ day ), LGZG-H (high dose of LGZG, at a dose of $22 \mathrm{~g} / \mathrm{kg} /$ day ) \and C176 (STING specific inhibitor, C-176, $6.7 \mathrm{mg} / \mathrm{kg} / \mathrm{day}$ ). Mice were fed with HFD and orally administered LGZG for an additional 9 weeks and then sacrificed for sampling. The mice body weight and fasting blood glucose (FBG) levels were measured weekly before sacrifice. 
Intra peritoneal glucose tolerance test (IPGTT) and insulin tolerance tests (ITTs) were performed at 3 day before sacrifice. For IPGTT, mice were fasted for $8 \mathrm{~h}$ and then intraperitoneally injected with glucose ( $2 \mathrm{mg} / \mathrm{g}$ body weight). The blood glucose levels were measured at $0,15,30,60,90$, and $120 \mathrm{~min}$ after glucose challenge respectively. For the insulin tolerance test (ITT), mice were fasted for $6 \mathrm{~h}$ and then intraperitoneal injected with human insulin $(0.75 \mathrm{U} / \mathrm{kg}$ body weight). The blood glucose level were measured at $0,30,45,60,90$ and 120 min after insulin injection respectively. At the end of experiment, blood samples for further assays were collected through abdominal heart puncture. The freshly separated liver tissues was quickly divided into several aliquots and stored in a $-80{ }^{\circ} \mathrm{C}$ refrigerator and $4 \%$ paraformaldehyde respectively for further use. Blood GGT, ALT, AST, TG and TC were measured by commercially available assay kits according to the standard manual.

\section{Histology}

Liver specimens were fixed in $4 \%$ paraformaldehyde, embedded in paraffin, and cut into $4 \mu \mathrm{m}$ thick sections. Sections were stained with hematoxylin and eosin $(\mathrm{H} \otimes \mathrm{E})$ according to a standard procedure for assessment histopathological changes. For Oil Red $O$ staining, The fixed liver specimens were embedded in optimum cutting temperature compound (OCT), and cut into $10 \mu \mathrm{m}$ thick sections. Sections were stained with $0.5 \%$ Oil Red 0 solution according to a standard procedure for assessment lipid accumulation. The degree of hepatic lipid deposition and oil red 0 positive area were quantified with Image J software.

\section{Immunofluorescence and Immunohistochemistry}

Paraffin-embedded mouse liver blocks from different groups were sliced into $4 \mu \mathrm{m}$-thick sections for immunofluorescence and immunohistochemistry staining. The antibodies against STING(1:200, CST, 16029T), F4/80 (1:200, CST,70076S),3-Nitrotyrosine (1: 100, Abcam, ab61392) , 4 Hydroxynonenal (1: 100 , Abcam, ab48506) were used according to manufacturer's protocol. The fluorescence intensity was analyzed by using Image $\mathrm{J}$ software and normalized by nuclei fluorescence intensity (6-diamidino-2phenylindole, DAPI, blue).

\section{Detection of mtDNA Content in Cytosolic Extracts by real time PCR(qPCR)}

Cytosolic mtDNA content was measured according to the previous work [21]. Briefly, freshly separated mice livers from different groups were divided into two aliquots. One aliquot was used to extract total DNA as the control for total mtDNA by using the DNA extraction kit according to the standard manual(Axygen US). The other one was used to extract cytosolic DNA. For the extraction of cytosolic DNA, the liver tissue was homogenized in $500 \mu \mathrm{L}$ buffer $(150 \mathrm{mM} \mathrm{NaCl}, 50 \mathrm{mM}$ Hepes, $25 \mu \mathrm{g} / \mathrm{mL}$ digitonin, $\mathrm{pH}$ 7.4) and then incubated for $10 \mathrm{~min}$ to permeabilize the plasma membrane. The homogenate was centrifuged $(980 \times \mathrm{g}, 5 \mathrm{~min})$ for 3 times to pellet intact cells. The cytosolic supernatants was further centrifuged at $17,000 \times \mathrm{g}$ for 25 min to pellet any remaining cellular debris, yielding cytosolic preparations free of nuclear, mitochondrial, and endoplasmic reticulum contamination. DNA was then isolated from these pure cytosolic fractions by using the DNA extraction kit according to the standard manual. 
Total RNA of liver tissues from sacrificed mice were prepared using TRIzol reagent. RNA $(1 \mu \mathrm{g})$ was reverse-transcribed into CDNA using First Strand cDNA Synthesis Kit according to the standard protocol. qPCR was performed on ABI 7500 qPCR systems according to the standard protocol. Data was analyzed using the $\triangle \triangle C T$ relative quantification method. Primers sequences and corresponding genes are shown in Table 1.

\section{Cell separation, culture and drug treatment}

Bone marrow cells were isolated from free-fed C57 mice and differentiated into bone-marrow-derived macrophages (BMDMs) as previously described [22] . Briefly, bone marrow cells were isolated from the tibias and femurs of the mice and cultured in DMEM containing 10\% fetal bovine serum and 20\% L929 culture supernatant for 6-8 days to obtain the BMDMs. To detect the effect of LGZG on the activation of STING mediate signal pathway, BMDMs were pretreated with $(7.5 \% \mathrm{NaHCO})$ or LGZG $\left(10^{-4} \mathrm{or} 10^{-3} \mathrm{~g} / \mathrm{mL}\right)$ for $2 \mathrm{~h}$ respectively as previously reported [19]. Then the cells were incubated with DMXAA $(75 \mu \mathrm{g} / \mathrm{mL})$ for $24 \mathrm{~h}$ in the absence or presence of LPS $(100 \mathrm{ng} / \mathrm{mL}$, dissolved in $1 \times$ phosphate buffered saline). After incubation, the cell lysates from different groups were harvest respectively for the detection of expression of STING and other related proteins. Furthermore, the cell culture supernatant from each group were collected for the detection of IFN $\beta$ and TNF by ELISA.

For HepG2 cells and macrophage co-culture study, the BMDMs treated with different doses LGZG (10-4 or $10^{-3} \mathrm{~g} / \mathrm{mL}$ )were respectively added to PA-stimulated HepG2 cells at a ratio of 1:10 and then incubated for another 48 hours and assayed for hepatocyte fat deposition and inflammatory responses as previously reported[13]. The lipid deposition was detected by Oil Red 0 staining. For the STING activation, cells were treated with DMXAA $(75 \mu \mathrm{g} / \mathrm{mL})$ at the last 24 hours. Details are refer to Luo et al.'s work[13].

\section{Immunoblotting}

For the immunoblotting, mice liver homogenates from and cultured cells were lysed with RIPA buffer. The denatured samples were loaded for SDS-PAGE, transferred, incubated with antibodies and visualized with enhanced chemiluminescence. The antibodies against F4/80, STING, TBK1, pTBK1, TNFa and tubulin were used. Ratios of p-TBK1 to TBK1 were normalized to Tubulin.

\section{Statistical analysis}

For cell studies, data are representative of three independent experiments. The Western blot images were semi quantified with the Image $J$ program. Statistical analysis of the data was performed using GraphPad Prism 7. Significance was assessed by performing an unpaired two-tailed Student's t test as indicated in individual figures. Quantitative data are presented as Mean \pm SEM. Statistical significance

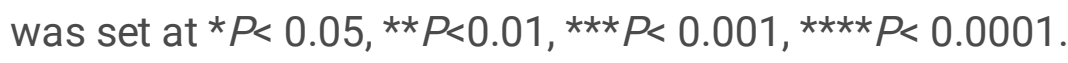

\section{Results}




\section{Identification of chemical compounds in LGZG by HPLC}

In the HPLC experiment, 4 compounds from FuLing, GuiZhi, BaiZhu and GanCao were detected in the LGZG(Fig.1a). The 4 compounds were tentatively characterized based on their formula and retention times. According to the Chinese Pharmacopoeia, Cinnamaldehyde, Glycyrrhizic acid, 2-Atractylenolide and Pachymic acid were used as references to verify the composition of LGZG. The representative HPLC chromatograms of standards and LGZG were shown in Fig. 1b, c. These results show that LGZG contains the characteristic peaks corresponding to 4 kinds of standard products.

\section{LGZG ameliorated HFD induced hepatic lipid metabolic disorder in mice}

To determine whether LGZG can alleviate HFD-induced hepatic lipid metabolic disorder, liver sections were subjected to pathological staining. The $H \otimes E$ and Oil-red $O$ staining results showed that LGZG treatment significantly reduced HFD-induced hepatic-lipid deposition (Fig. 2a-b). The hepatic steatosis rate of the HFD nice model group was up to $50 \%$, while after LGZG treatment, it reduced to about $10 \%$ ( Fig. 2c). Interestingly, the STING specific inhibitor $\mathrm{C} 176$ can also significantly reduce the hepatic lipid deposition in HFD mice with a hepatic steatosis rate of about 27\%(Fig. 2c-d). Furthermore, LGZG significantly decreased the blood TC level but did not affect TG in the HFD mice (Fig. 2e). In addition, LGZG can significantly reduce the blood GGT, ALT and AST to ease HFD-induced liver damage (Fig. 2f-g). These results suggest that both LGZG and STING-specific blocker ameliorate HFD induced hepatic lipid metabolism disorder in mice.

\section{LGZG reduced HFD induced insulin resistance in mice}

HFD induces obesity. We firstly tested the effect of LGZG on the body weight of HFD mice. The results revealed that LGZG had little effect on HFD induced body weight increase (Fig. 3a). However, LGZG can significantly inhibit HFD-induced hyperglycemia in a dose dependent manner (Fig. 3b). IR is closely related to liver inflammation and lipid metabolism [5]. To detected whether LGZG could reduce HFD induced IR, we further tested the effect of LGZG on glucose tolerance and IR in HFD mice. IPGTT results showed that HFD induced significant glucose tolerance ( $48.46 \pm 2.48$ vs $90.44 \pm 6.89$, con vs. mod, Fig. $3 \mathrm{~b}$ ) and IR $(12.42 \pm 1.19$ vs $26.09 \pm 2.33$, con vs. mod, Fig. $3 c)$ in mice. The blood glucose levels in all groups peaked at 15 minutes after intra peritoneal glucose loading and then decreased. Compared with the model group, the blood glucose of HFD mice in the LGZG treatment group decreased rapidly after glucose loading, but the blood glucose of the model group (model) remained at a higher level(Fig. 3c). The ITT results revealed that after insulin injection, the blood glucose of HFD mice in the LGZG treatment group dropped rapidly in dose dependent manner, but the blood glucose of the model group had little change (Fig. 3d). The AUC of both GTT and ITT in the high dose of LGZG group was significantly lower than that in the model group ( $p<0.001$, Fig. 3e, 3f). However, STING-specific blocker had little effect on HFD induced glucose tolerance and IR in mice. These results suggest that LGZG ameliorate HFD induced glucose tolerance and IR in mice. Furthermore, HFD induced glucose tolerance and insulin resistance might not be STING dependent. 


\section{LGZG alleviated hepatic mitochondrial damage and oxidative stress in HFD-fed mice}

HFD induced obesity and IR can cause mitochondrial damage, which result in the release of oxidative free radicals as well as oxidative stress (OS). Increased OS can in turn exacerbate mitochondrial damage. 8OHdG, 4-HNE and 3-NT are three sensitive biomarker for OS. To detect the effect of LGZG on HFD induced OS, we compared the expression of 8-OHdG, 4-HNE and 3-NT in the liver from different groups of HFD mice. The results of immunohistochemistry and immunofluorescence showed that HFD significantly increased the expression of 8-OHdG, 4-HNE and 3-NT whereas LGZG significantly reduced their expression in liver (Fig. 4a-C). OS further aggravates mitochondrial damage and the release of the mitochondrial DNA (mtDNA). To detect mtDNA copy number in the cytosol, we purified total DNA from the cytosolic fraction of hepatocyte freshly isolated from mice of different group. The purity of the cytosolic fraction was confirmed by cell-fractionation studies, which showed no contamination with the nuclear markers and Tert (Fig. 4d). The Tert gene primer pairs were used to detect the nuclear DNA while the primers of Dloop1 to 3, mtND4, and Cytb were used to detect mtDNA. The qPCR result revealed that mtDNA levels were significantly decreased in the cytosol of hepatocyte from LGZG groups compared with model group (Fig. 4e). These results suggest that LGZG could significantly reduce HFD-induced mitochondrial damage and OS.

\section{LGZG reduced liver macrophage infiltration and STING expression in HFD-fed mice.}

HFD-induced mitochondrial damage and OS in the liver can lead to the release of mtDNA[14]. While the released mtDNA can be swallowed by hepatic-macrophages (Kupffer cells), which activates STINGmediated pathways and induces hepatic-inflammation [14,23]. To detect the effect of LGZG on the expression of STING, we test the expression of STING in liver from different groups of HFD fed mice. Immunofluorescence results showed that compared with normal liver (control), Kupffer cells(F4/80 positive cells, $\mathrm{F} 4 / 80^{+}$, green) in model group increased significantly (Fig. 5a). Furthermore, STING mainly expressed in Kupffer cells(STING positive and F4/80 positive cells, $\mathrm{STING}^{+} \mathrm{F} 4 / 80^{+}$cells), and its expression was also increased in model group (Fig. 5a-b). However, after LGZG treatment, STING ${ }^{+} \mathrm{F} 4 / 80^{+}$ cells in the liver of HFD mice are significantly reduced (Fig. 5b, 5c). These results suggest that LGZG reduces liver macrophage infiltration and STING expression in HFD-fed mice. Furthermore, these results are consistent with previous work that the hepatic macrophages are the key cellular sources of STING in the liver[23].

\section{LGZG inhibited the activation of STING-mediated signaling pathways in BMDMs(Bone marrow-derived macrophages)}

Studies have shown that STING-mediated signaling pathways such as STING-TBK1-NF-KB pathway play important roles in regulating the pro-inflammatory response of macrophages[24, 25]. The STING activator DMXAA significantly enhanced the LPS-induced inflammatory response in macrophages, which result in the increasing of the release of IFN $\beta$ and the phosphorylation of JNK p46 and NFKB p65[26]. To detect whether LGZG can regulate STING-mediated signal pathway in macrophages and inhibit macrophages 
induced inflammation, we tested the effect of LGZG on the STING-TBK1-NF-KB signaling pathway in mice BMDMs. The ELISA results showed that the DMXAA and LPS significantly increased the release of IFN $\beta$ and TNFa while LGZG dose dependently inhibited DMXAA and LPS induced release of these cytokines (Fig. 6a-b). Furthermore, DMXAA and LPS up-regulated the expression of STING and the phosphorylation of TBK1 whereas LGZG dose dependently inhibited this upregulation in BMDMs (Fig. 6c-e). These results indicate that LGZG can directly inhibit the activation of the STING-TBK1-NF-KB pathway in macrophages and reduce the release of inflammatory factors.

\section{BMDM treated with LGZG reduced lipid deposition in PA-induced high-lipid cell model by inhibiting STING- mediated pathway}

STING-driven macrophages have been reported to enhance hepatocyte fat deposition and proinflammatory responses $[13,23]$. To recapitulate our in vivo findings and gain mechanistic insights, LGZG treated or untreated BMDMs (LGZG-BMDM or BMDM) were co-incubated with PA-stimulated HepG2 cells and assayed for hepatocyte fat deposition. The Oil Red $O$ staining results showed that the cell lipid deposition in BMDM-HepG2 co-culture group was significantly increased after DMXAA was added (Fig. $7 a-b)$. However, after adding different doses of LGZG $\left(10^{-4} \mathrm{~g} / \mathrm{mL}, \mathrm{L}^{-4}\right.$ or $\left.10^{-3} \mathrm{~g} / \mathrm{mL}, \mathrm{L}^{-3}\right)$ treated BMDM to DMXAA and PA-stimulated HepG2 cells (DMXAA+BMDM+HepG $2+L^{-3}$ or DMXAA+BMDM+HepG $2+L^{-4}$ ), the cell lipid deposition Significantly reduced. These results suggest that STING activation enables macrophages to generate factors that can enhance hepatocyte lipid deposition while LGZG can reduce cell lipid deposition induced by STING activation in macrophages.

\section{Discussion}

Previous studies have shown that hepatic macrophages-mediated inflammation is one of the key factors leading to the progression of NAFLD and other metabolic disorders [5]. These provide possibilities for the promotion of the application of TCM containing anti-inflammatory component in the prevention and treatment of metabolic diseases. LGZG is widely used in the treatment of metabolic diseases such as NAFLD and Type 2 diabetes. Although LGZG has been reported to alleviate NAFLD via reducing OS, BetaOxidation and cholesterol secretion $[18,27]$, whether LGZG can alleviate NAFLD by inhibiting hepatic macrophage-mediated inflammation is not yet fully understood. Here we demonstrated that LGZG could significantly reduce the mitochondrial damage and OS in the liver of HFD fed mice. Furthermore, LGZG can inhibit inflammation mediated by STING activation in hepatic-macrophages, thereby alleviating HFDinduced liver lipid deposition.

STING, which closely related to inflammation, is highly expressed in hepatic macrophages (Kupffer cells) in both NAFLD patients and HFD-mice models[13]. The inflammatory response caused by activation of the STING-TBK1-NF-KB pathway in Kupffer cells promotes the progression of NAFLD[14]. mtDNA released by mitochondrial damage is one of the key ways to activate the STING- mediated pathway in hepatic macrophages[14]. The activation of STING-TBK1-NF-KB pathway can promote the release of a large number of inflammatory cytokines, including IFN $\beta$ and TNFa. Increased TNFa-producing hepatic 
macrophages are crucial for triggering NASH via monocyte recruitment[28]. IFN $\beta$ and TNFa released by hepatic macrophages not only aggravate mitochondrial damage and OS in hepatocytes, but also directly activate the JNK-NF-KB pathway in hepatocytes, thereby promoting lipid deposition[13]. These can explain why the inhibition of STING activation elicited by LGZG and STING specific inhibitor in hepaticmacrophages can reduce HFD induced liver lipid deposition.

LGZG contains a lot of anti-inflammatory components, such as tuckahoe polysaccharides, atractylodes polysaccharides which may down regulate the inducible nitric oxide synthase (iNOS)[29]. iNOS is controlled through inducible transcription in response to proinflammatory cytokines, such as interferon gamma (IFN- $\gamma)$, TNF-a, and IL-1 $\beta[30]$. These may explain why LGZG could directly inhibit the LPS or STING specific agonist induced activation of STING-TBK1-NF-KB pathway in BMDMs. Furthermore, previous study showed that STING mediate neuro-inflammatory injury by suppressing AMPK pathway[31]. Therefore, another explanation is that some components in LGZG may activate the AMPK pathway, thereby inhibiting the inflammation induced by STING activation. Both of these two explanations are reasonable, which is consistent with the complex composition and multiple targets of LGZG. However, more investigation is need to illustrate the mechanism, which is our ongoing work.

Insulin resistance is another key factor for the progression of NAFLD, which is closely related to inflammation [5]. However, our results showed that LGZG can significantly improve HFD-induced glucose resistance and insulin resistance in mice, but the STING inhibitor C176 has little effect on insulin resistance and glucose resistance, which is consistent with previous work that diet-induced adipose insulin resistance and glucose intolerance is related to STING signaling but not dependent on it [32]. In contrast, the STING-mediated pathway in macrophages is more closely related to HFD-induced lipid deposition in hepatocytes.

\section{Conclusions}

In summary, this study demonstrates that LGZG can alleviate HFD-induced hepatic steatosis through inhibiting the activation of STING in macrophages, which provides novel insight for elucidating the molecular mechanism of LGZG's anti-NAFLD effect.

\section{Abbreviations}

LGZG Lingguizhugan decoction

NAFLD Non-alcoholic fatty liver disease

HFD high fat diet

STING stimulator of IFN genes

BMDMs bone-marrow-derived macrophages 
DMXAA 5,6-dimethylxanthenone-4-acetic acid

IR insulin resistance

LPS lipopolysaccharides

TBK1 TANK-binding kinase 1

IFN $\beta \quad$ interferon beta

TNFa tumor necrosis factor alpha

$\mathrm{NASH}$ nonalcoholic steatohepatitis

HCC hepatocellular carcinoma

TCM Traditional Chinese Medicine

PPPM perspective of predictive, preventive and personalized medicine

TMEM173 transmembrane protein 173

cGAMP cyclic guanosine phosphate-adenosine phosphate

IRF3 IFN regulatory factor 3

PC Poria cocos

RC Ramulus Cinnamomi

RAM Rhizoma Atractylodis Macrocephalae

RG Radix Glycyrrhizae

HPLC high-performance liquid chromatography

FBG fasting blood glucose

IPGTT intra peritoneal glucose tolerance test

ITT insulin tolerance test

GGT gamma glutamyl transferase

ALT alanine aminotransferase

AST aspartate aminotransferase 


$\begin{array}{ll}\text { TG } & \text { triglyceride } \\ \text { TC } & \text { total cholesterol } \\ \text { H囚E } & \text { hematoxylin and eosin } \\ \text { OCT } & \text { optimum cutting temperature compound } \\ \text { 3NT } & \text { 3-Nitrotyrosine } \\ \text { 4HNE } & \text { 4 Hydroxynonenal } \\ \text { DAPI } & \text { 6-diamidino-2-phenylindole } \\ \text { 8-OHdG } & \text { 8-Hydroxy-2'-deoxyguanosine } \\ \text { PA } & \text { palmitate } \\ \text { ROS } & \text { reactive oxygen species }\end{array}$

\section{Declarations}

\section{Acknowledgements}

Not applicable.

\section{Authors' contributions}

Lin Cao, Erjin Xu and Rendong Zheng designed the study and wrote the manuscript. Lin Cao, Erjin Xu, Juan Ye, Hongping, Sun Yaofu Fan, Yu Chen and Yijiao Xu, performed the animal and molecular biology experiment. Fei Huang, Shaofeng Xie, Zhili ZhangChen, Jing Cao participated in the coordination and the analyzing of data. Wen Cao and Chao Liu critically revised the manuscript. All authors have read and agreed to submit the manuscript.

\section{Funding}

This study was supported by the National Nature Science Foundation of China(NNSFC) 81804070(to Lin Cao), and research program of Suzhou Municipal Science and Technology Bureau SYSD2019148 (to Fei Huang).

\section{Availability of data and materials}

The data used to support the findings of this study are available from the corresponding author upon request. 
All procedures were performed in accordance with the guidelines of the

Nanjing University of Chinese Medicine ethics committee.

\section{Consent for publication}

Not applicable.

\section{Competing interests}

The authors declare that they have no competing interests.

\section{Author Details}

1. Affiliated Hospital of Integrated Traditional Chinese and Western Medicine, Nanjing University of Chinese Medicine, No.100, Shizi Street, Hongshan Road, Nanjing 210028, China

2. Jiangsu Province Academy of Traditional Chinese Medicine, No.100, Shizi Street, Hongshan Road, Nanjing 210028, China

3. Suzhou Traditional Chinese Medicine Hospital Affiliated to Nanjing University of Chinese Medicine, No.18 Yangsu Road, Gusu District, Suzhou 215002, China

4. Affiliated Hospital of Nanjing University of Chinese Medicine,155 Hanzhong Road, Qinhuai District, Nanjing 210029, China

5. The Affiliated Jiangning Hospital of Nanjing Medical University, NO.169, Dongshan street, Hushan Road, Jiangning District, Nanjing211100, China

\section{References}

1. Huang TD, Behary J, Zekry A. Non-alcoholic fatty liver disease: a review of epidemiology, risk factors, diagnosis and management. Internal medicine journal. 2020;50(9):1038-47.

2. Cotter TG, Rinella M. Nonalcoholic Fatty Liver Disease 2020: The State of the Disease. Gastroenterology. 2020;158(7):1851-64.

3. Gehrke N, Schattenberg JM. Metabolic Inflammation-A Role for Hepatic Inflammatory Pathways as Drivers of Comorbidities in Nonalcoholic Fatty Liver Disease. 2020;Gastroenterology. 158 (7): 19291947.

4. Matulewicz N, Karczewska-Kupczewska M. Insulin resistance and chronic inflammation. Postepy higieny i medycyny doswiadczalnej (Online). 2016;70(0):1245-58.

5. Shimobayashi M, Albert V, Woelnerhanssen B, Frei IC, Weissenberger D, Meyer-Gerspach AC, et al. Insulin resistance causes inflammation in adipose tissue. The Journal of clinical investigation. 2018;128(4):1538-50.

6. He L, Liu X, Wang L, Yang Z. Thiazolidinediones for nonalcoholic steatohepatitis: A meta-analysis of randomized clinical trials. Medicine. 2016;95(42):e4947. 
7. Chu JW, Abbasi F, Lamendola C, McLaughlin T, Reaven GM, Tsao PS. Effect of rosiglitazone treatment on circulating vascular and inflammatory markers in insulin-resistant subjects. Diabetes \& vascular disease research. 2005;2(1):37-41.

8. Lan W, Wang Z, Liu J, Liu H. Methionyl-Methionine Exerts Anti-Inflammatory Effects through the JAK2-STAT5-NF-KB and MAPK Signaling Pathways in Bovine Mammary Epithelial Cells. Journal of agricultural and food chemistry. 2020;68(47):13742-50.

9. Barber GN. STING: infection, inflammation and cancer. Nature Reviews Immunology. 2015;15(12):760-70.

10. Zhang X, Wu J, Liu Q, Li X, Li S, Chen J, et al. mtDNA-STING pathway promotes necroptosisdependent enterocyte injury in intestinal ischemia reperfusion. Cell Death \& Disease. 2020;11(12):1050.

11. Hu HQ, Qiao JT, Liu FQ, Wang JB, Sha S, He Q, et al. The STING-IRF3 pathway is involved in lipotoxic injury of pancreatic $\beta$ cells in type 2 diabetes. Molecular and cellular endocrinology. 2020;518:110890.

12. Bai J, Liu F. The cGAS-cGAMP-STING Pathway: A Molecular Link Between Immunity and Metabolism. Diabetes. 2019;68(6):1099-108.

13. Luo X, Li H, Ma L, Zhou J, Guo X, Woo S-L, et al. Expression of STING Is Increased in Liver Tissues From Patients With NAFLD and Promotes Macrophage-Mediated Hepatic Inflammation and Fibrosis in Mice. Gastroenterology. 2018;155(6):1971-84.

14. Yu Y, Liu Y, An W, Song J, Zhang Y, Zhao X. STING-mediated inflammation in Kupffer cells contributes to progression of nonalcoholic steatohepatitis. The Journal of clinical investigation. 2019;129(2):546-55.

15. Xu J, Wang R, You S, Zhang L, Zheng P, Ji G, et al. Traditional Chinese medicine Lingguizhugan decoction treating non-alcoholic fatty liver disease with spleen-yang deficiency pattern: Study protocol for a multicenter randomized controlled trial. Trials. 2020;21(1):512.

16. Zhu M, Hao S, Liu T, Yang L, Zheng P, Zhang L, et al. Lingguizhugan decoction improves nonalcoholic fatty liver disease by altering insulin resistance and lipid metabolism related genes: a whole trancriptome study by RNA-Seq. Oncotarget.2017;8(47): 82621-82631

17. Yang L, Lin W, Nugent CA, Hao S, Song H, Liu T, et al. Lingguizhugan Decoction Protects against High-Fat-Diet-Induced Nonalcoholic Fatty Liver Disease by Alleviating Oxidative Stress and Activating Cholesterol Secretion. International Journal of Genomics. 2017;2017(9):1-12.

18. Liu T, Yang LL, Zou L, Li DF, Wen HZ, Zheng PY, et al. Chinese Medicine Formula Lingguizhugan Decoction Improves Beta-Oxidation and Metabolism of Fatty Acid in High-Fat-Diet-Induced Rat Model of Fatty Liver Disease. Evidence-based Complementary \& Alternative Medicine (eCAM). 2013;2013:429738

19. Xi F, Sang F, Zhou C, Ling Y. Protective effects of Lingguizhugan decoction on amyloid-beta peptide (25-35)-induced cell injury: Anti-inflammatory effects. Neural regeneration research. 2012;7(36):286773. 
20. Hua D, Yang J, Meng Q, Ling Y, Wei Q, Wang Z, et al. Soufeng sanjie formula alleviates collageninduced arthritis in mice by inhibiting Th17 cell differentiation. Chinese medicine. 2021;16(1):39.

21. Bai J, Cervantes C, Liu J, He S, Zhou H, Zhang B, et al. DsbA-L prevents obesity-induced inflammation and insulin resistance by suppressing the mtDNA release-activated cGAS-cGAMP-STING pathway. Proc Natl Acad Sci U S A. 2017;114(46):12196-201.

22. Xu H, Li H, Woo SL, Kim SM, Shende VR, Neuendorff N, et al. Myeloid cell-specific disruption of Period1 and Period2 exacerbates diet-induced inflammation and insulin resistance. The Journal of biological chemistry. 2014;289(23):16374-88.

23. Maher JJ. Macrophages Steal STING From the Infectious Disease Playbook to Promote Nonalcoholic Fatty Liver Disease. Gastroenterology. 2018;155(6):1687-8.

24. Wang X, Rao H, Zhao J, Wee A, Li X, Fei R, et al. STING expression in monocyte-derived macrophages is associated with the progression of liver inflammation and fibrosis in patients with nonalcoholic fatty liver disease. Laboratory Investigation. 2020;100(4):542-52.

25. Ahn J, Barber GN. STING signaling and host defense against microbial infection. Experimental \& Molecular Medicine. 2019;51(12):1-10.

26. Downey CM, Aghaei M, Schwendener RA, Jirik FR. DMXAA causes tumor site-specific vascular disruption in murine non-small cell lung cancer, and like the endogenous non-canonical cyclic dinucleotide STING agonist, 2'3'-cGAMP, induces M2 macrophage repolarization. PloS one. 2014;9(6):e99988.

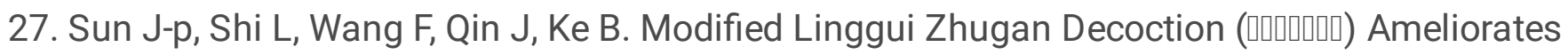
Glycolipid Metabolism and Inflammation via PI3K-Akt/mTOR-S6K1/AMPK-PGC-1a Signaling Pathways in Obese Type 2 Diabetic Rats. Chinese Journal of Integrative Medicine. 2020.

28. Tosello-Trampont AC, Landes SG, Nguyen V, Novobrantseva TI, Hahn YS. Kuppfer cells trigger nonalcoholic steatohepatitis development in diet-induced mouse model through tumor necrosis factor-a production. The Journal of biological chemistry. 2012;287(48):40161-72.

29. Guo L, Ma R, Sun H, Raza A, Tang J, Li Z. Anti-Inflammatory Activities and Related Mechanism of Polysaccharides Isolated from Sargentodoxa cuneata. Chemistry \& biodiversity. 2018;15(11):e1800343.

30. Jiang G-L, Yang X-L, Zhou H-J, Long J, Liu B, Zhang L-M, et al. cGAS knockdown promotes microglial M2 polarization to alleviate neuroinflammation by inhibiting cGAS-STING signaling pathway in cerebral ischemic stroke. Brain Research Bulletin. 2021;171:183-95.

31. Peng $Y$, Zhuang J, Ying G, Zeng H, Zhou H, Cao Y, et al. Stimulator of IFN genes mediates neuroinflammatory injury by suppressing AMPK signal in experimental subarachnoid hemorrhage. Journal of neuroinflammation. 2020;17(1):165.

32. Mao Y, Luo W, Zhang L, Wu W, Yuan L, Xu H, et al. STING-IRF3 Triggers Endothelial Inflammation in Response to Free Fatty Acid-Induced Mitochondrial Damage in Diet-Induced Obesity. Arteriosclerosis, thrombosis, and vascular biology. 2017;37(5):920-9. 


\section{Tables}

Table 1 Primer pair sequences

\begin{tabular}{llll} 
Gene & Accession no. & Primer & Sequence5'-3' \\
\hline B-Actin & NM_007393.5 & Forward & GTTGGTTGGAGCAAACATC \\
& & Reverse & CTTATTTCATGGATACTTGGAATG \\
\hline Tert & NC_000079.6 & Forward & CTAGCTCATGTGTCAAGACCCTCTT \\
& & Reverse & GCCAGCACGTTTCTCTCGTT \\
mtDNA loop 1 & NC_005089 & Forward & AATCTACCATCCTCCGTGAAACC \\
mtDNA loop 2 & & Reverse & TCAGTTTAGCTACCCCCAAGTTTAA \\
mtDNA loop 3 & NC_005089 & Forward & CCCTTCCCCATTTGGTCT \\
& & Reverse & TGGTTTCACGGAGGATGG \\
& NC_005089 & Forward & TCCTCCGTGAAACCAACAA \\
& & Reverse & AGCGAGAAGAGGGGCATT
\end{tabular}

\section{Figures}


a

Gui Zhi
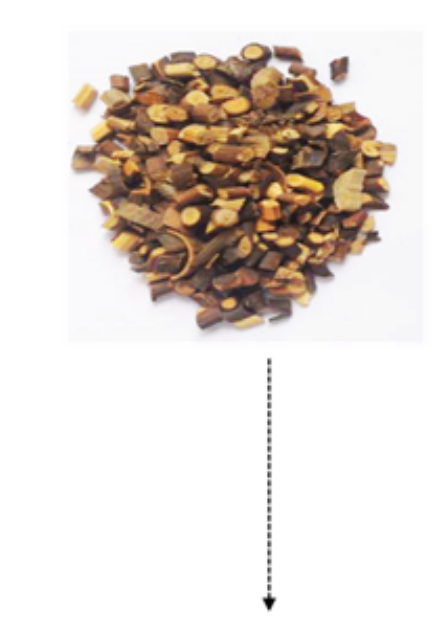

b

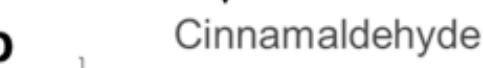

$$
\text { Zhi Gan Cao }
$$

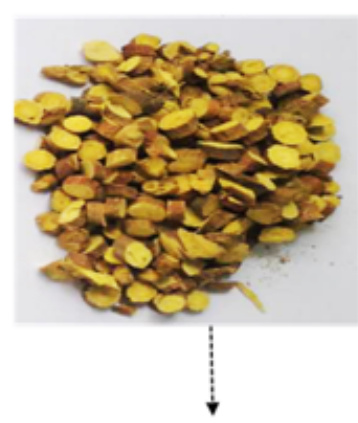

Glycyrrhizic acid

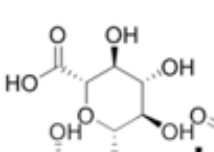

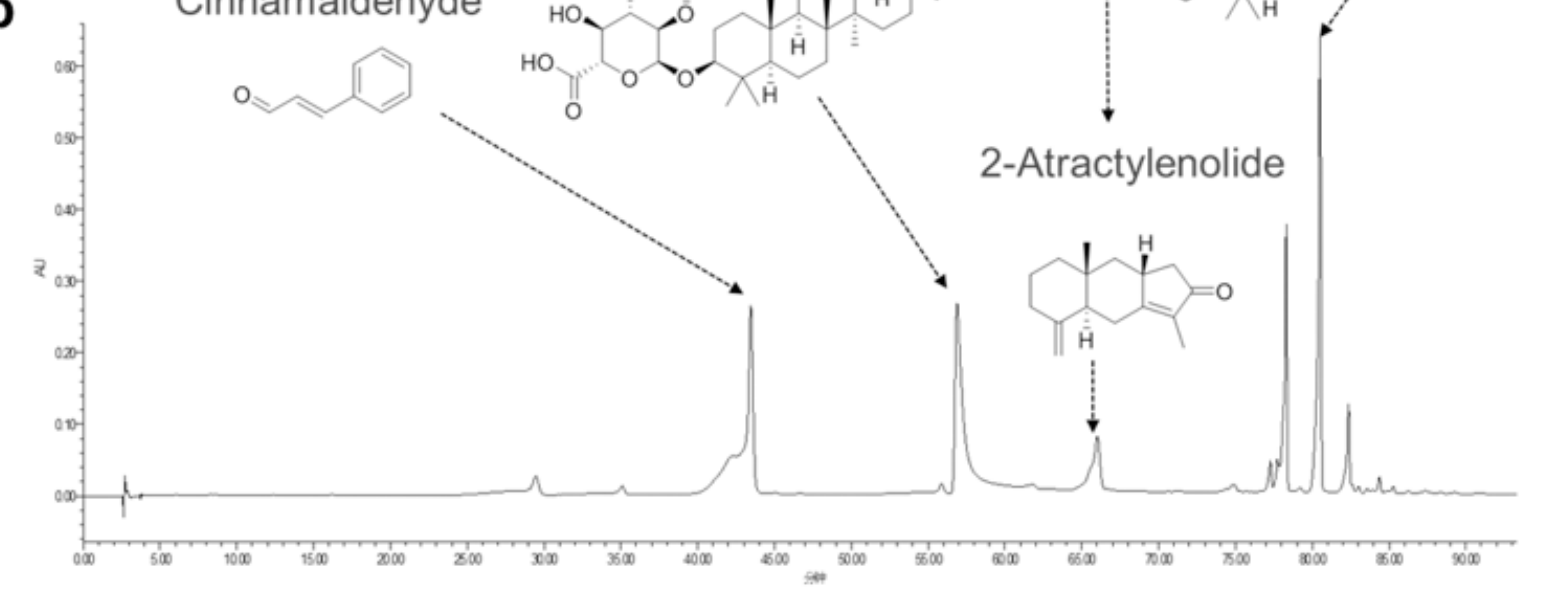

Chao Bai Zhu
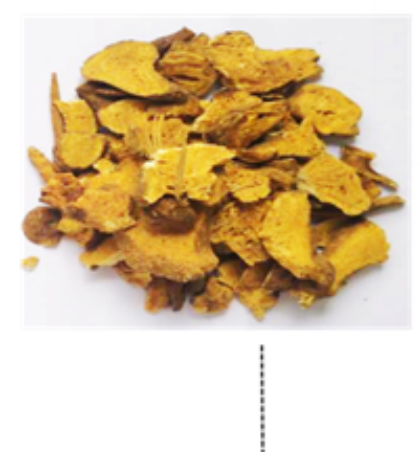

Fu Ling

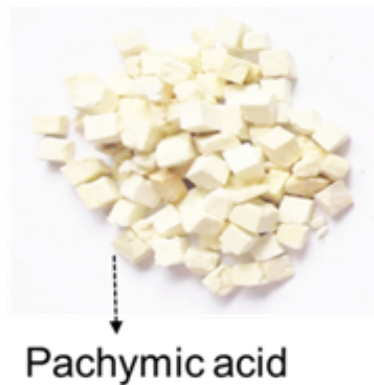

Pachymic acid

C

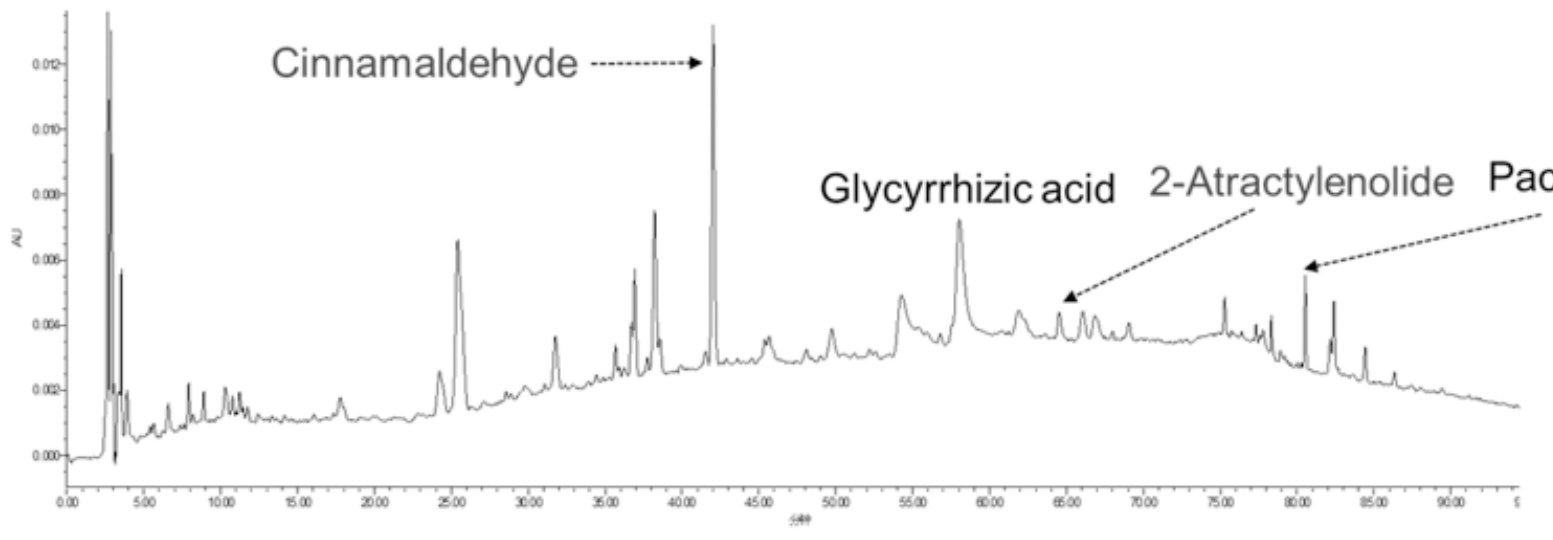

\section{Figure 1}

Identification of chemical compounds in LGZG by HPLC a Images represent the four components that make up LGZG. b HPLC chromatograms of the mixture of Cinnamaldehyde, Glycyrrhizic acid, 2Atractylenolide and Pachymic acid at $237 \mathrm{~nm}$. c HPLC chromatograms of the LGZG at $237 \mathrm{~nm}$. 

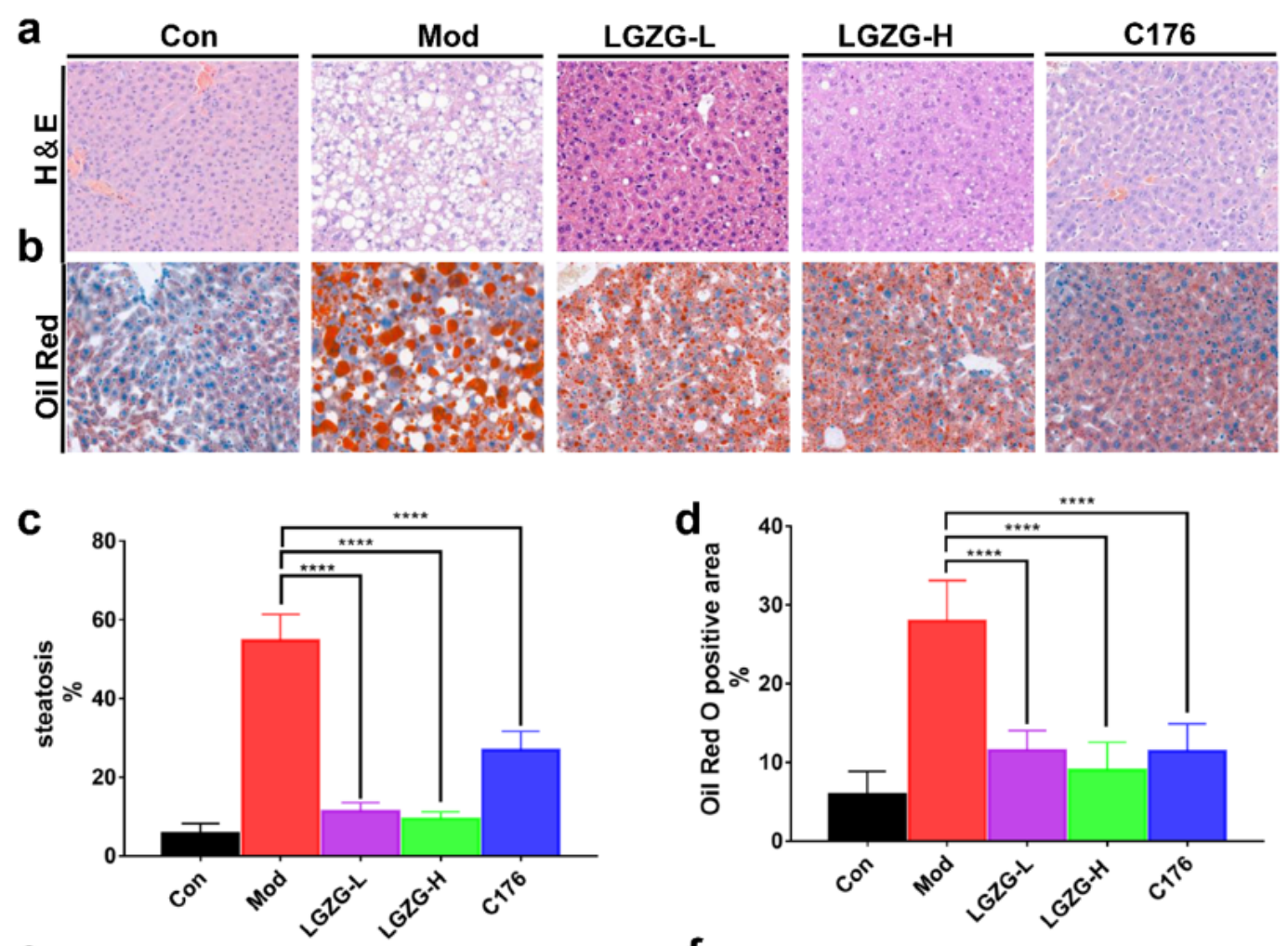

e

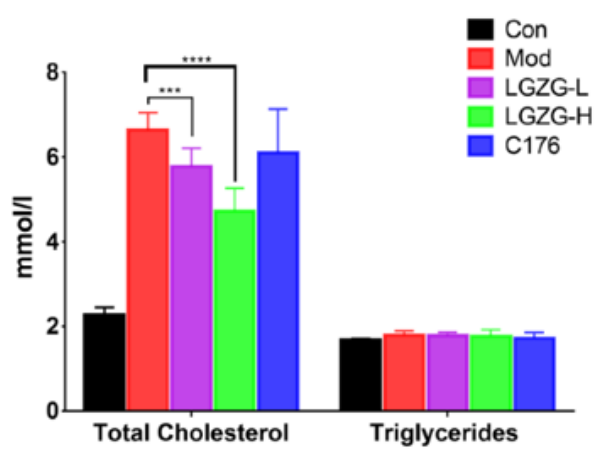

f
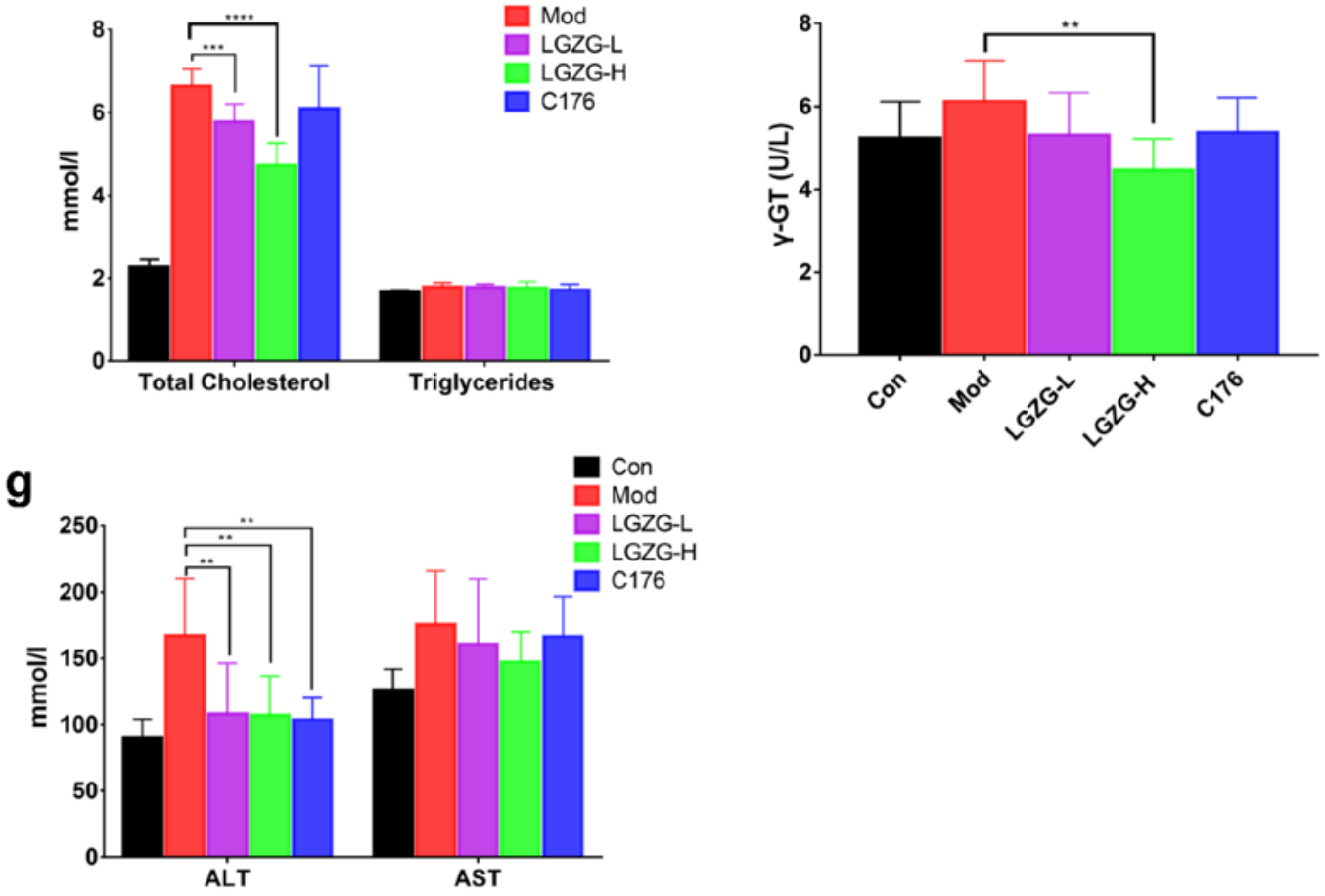

Figure 2

Effects of LGZG on HFD induced hepatic lipid metabolic disorder in mice. a, b Representative H\&Estained(a) and oil red stained images(b) of liver tissues from control and treated mice. c, d Quantification of steatosis area(c) and oil red positive area(d). e-g The blood concentrations of total cholesterol (TC) , triglyceride (TG, e) , gamma glutamyl transferase (GGT) (f), alanine aminotransferase (ALT, g) and 
aspartate aminotransferase (AST, g). The data are expressed as mean $\pm S D(n=9) . * P<0.05, * \star P<0.01$, *** $P<0.001$, $* \star * * ~ P<0.001$ vs. Mod group. The white scale bars represent $50 \mu \mathrm{m}$.
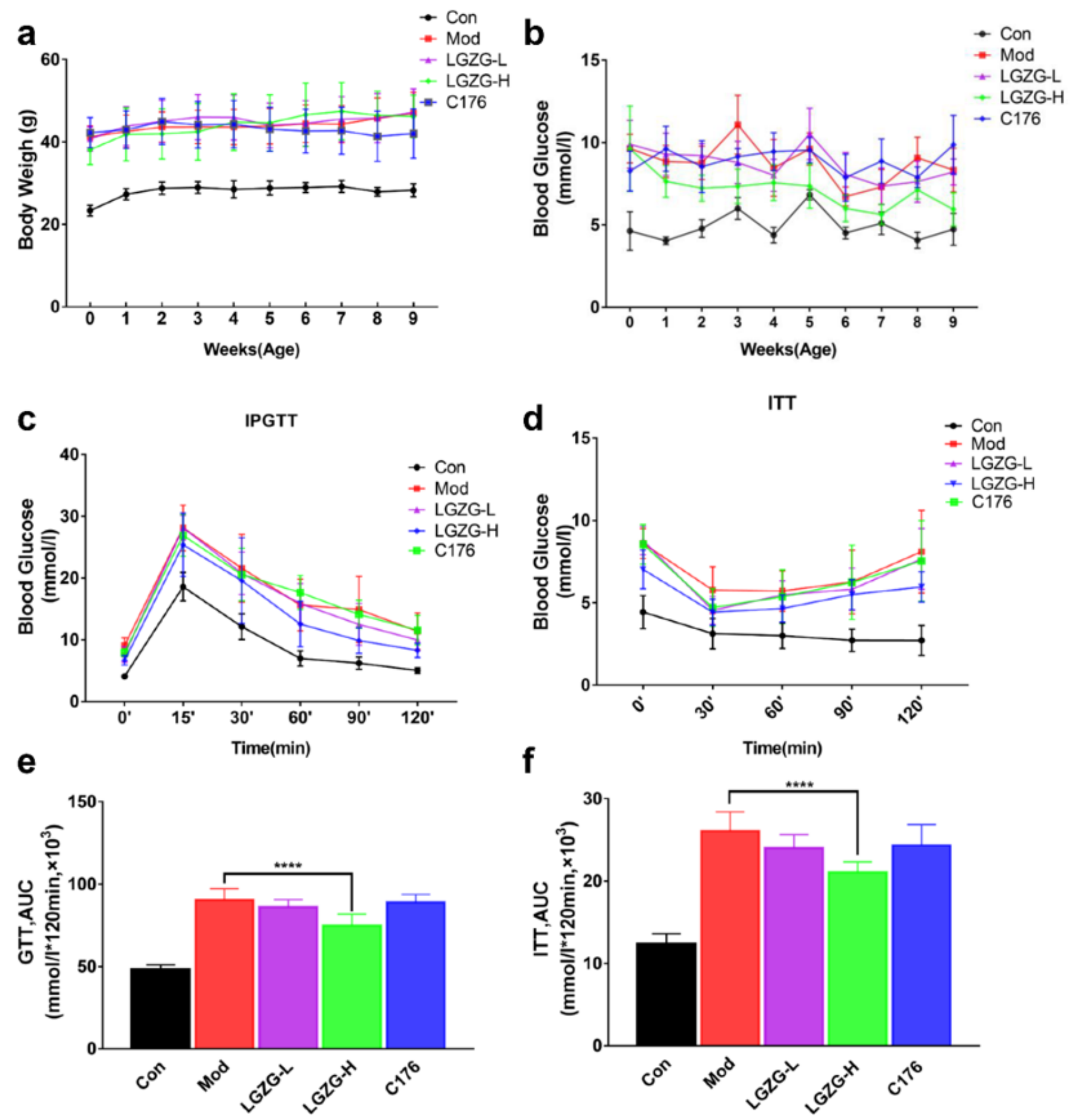

Figure 3

Effects of LGZG on hyperglycemia, glucose tolerance and insulin tolerance in HFD-fed mice. a Body weight changes during the LGZG treated period. Weight is measured once a week ( $n=9$ per group). $b$ Changes in fasting blood glucose levels after a switch to HFD The blood glucose level is measured once 
a week. c, d Glucose tolerance (c) and insulin tolerance (d) after 9 weeks of LGZG treatement on HFD. For the IPGTT, mice were injected with glucose ( $2 \mathrm{mg} / \mathrm{g}$ body weight) after an 8-hour fastting. e, $\mathrm{f}$ AUC of the IPGTT(e) and ITT(f). For the ITT, mice were injected with insulin ( $0.75 \mathrm{U} / \mathrm{kg}$ body weight) after a 6-hour

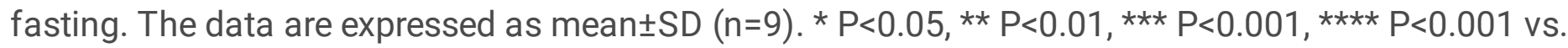
Mod group.

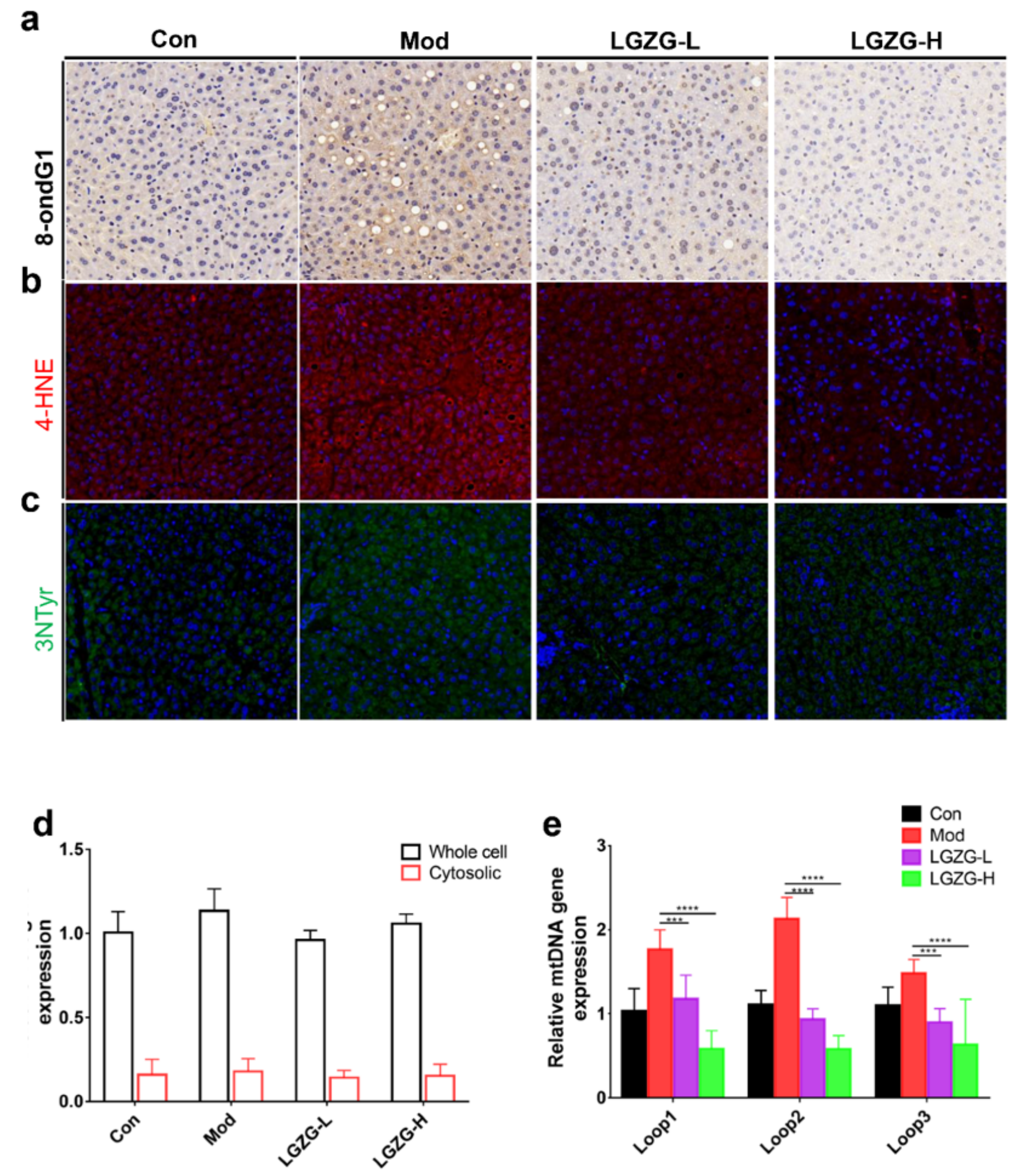

Figure 4 
Effects of LGZG on liver mitochondrial damage and oxidative stress in HFD-fed mice. a-c Representative Immunohistochemistry and Immunofluorescence images of liver sections from control and treated mice. For the staining of the expression of oxidative stress related markers, Livers sections were immunestained with antibodies against 8-OHdG(a), 4HNE(b) and 3-NT(c). d, e The relative expression of Nuclearencoded Tert gene(d) and Cytosolic mtDNA content(e) in mice livers from control and treated mice. The relative expression of these genes were detected by qPCR and calculated with $2-\triangle \triangle C T$. The data are expressed as mean $\pm S D(n=9)$. ${ }^{*} P<0.05, * \star P<0.01$, ${ }^{\star \star \star} P<0.001$, $* \star \star \star ~ P<0.001$ vs. Mod group. The white scale bars represent $50 \mu \mathrm{m}$.
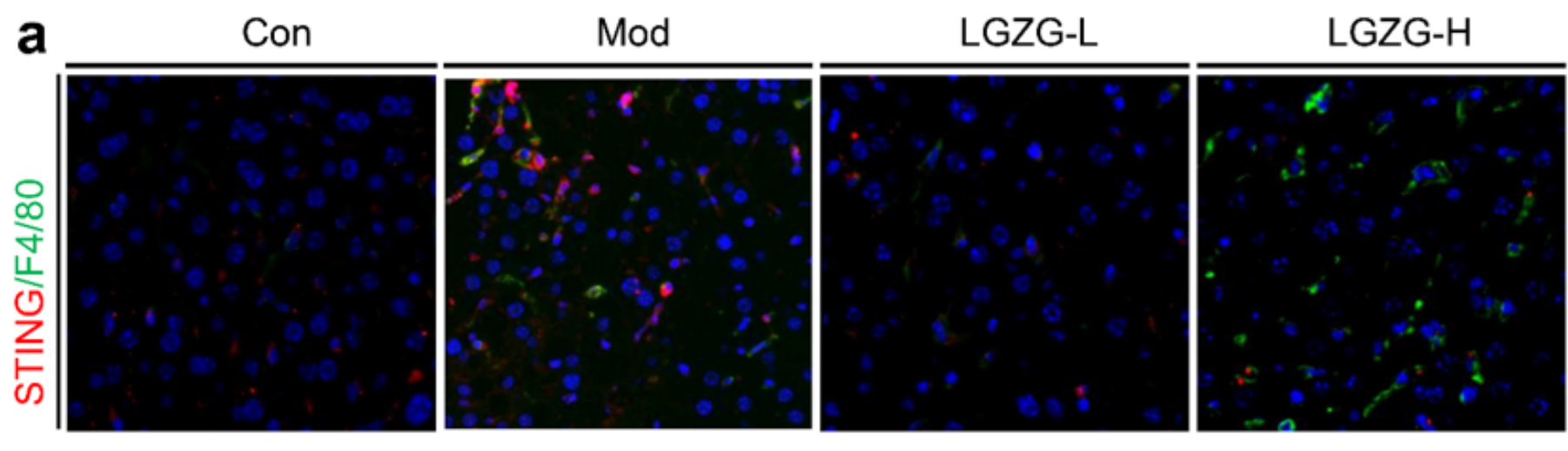

b
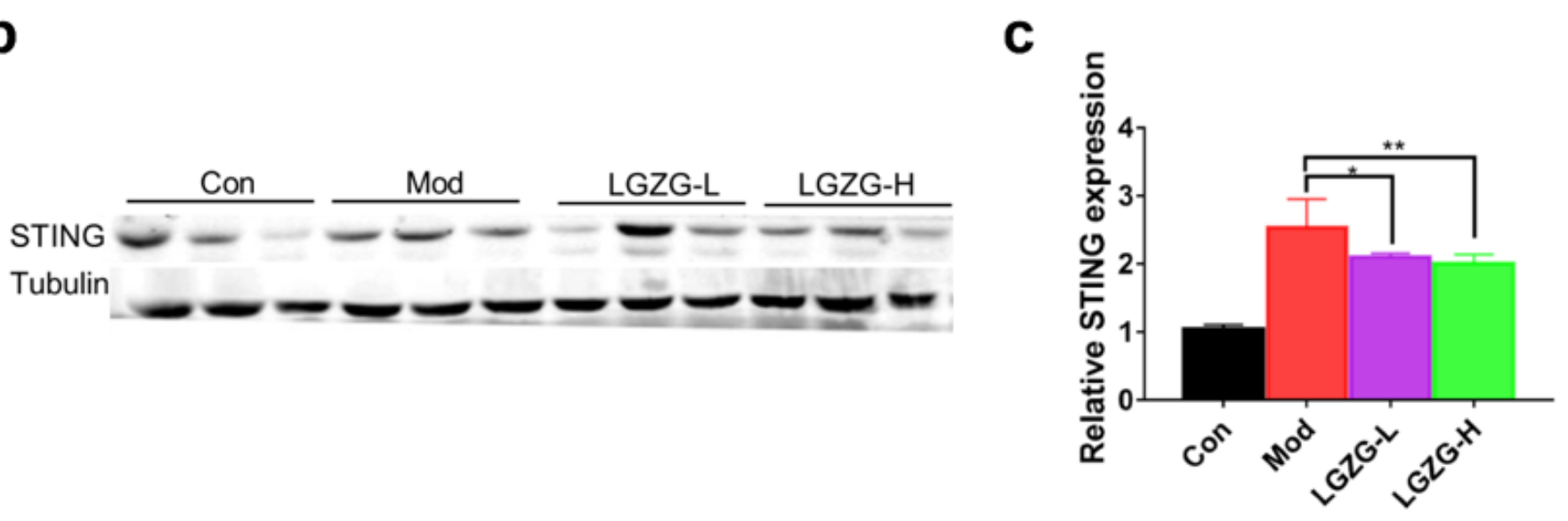

\section{Figure 5}

Effects of LGZG on liver macrophage infiltration and STING expression in HFD-fed mice. a Representative Immunofluorescence images of liver sections from control and treated mice. For the staining of STING and F4/80, Liver sections were double-immunostained with antibodies against STING and F4/80. The merged images including staining of STING (red), F4/80 (green), nuclei (blue) shown in(a). b, c Immunoblotting analysis of the expression of STING in livers from control and treated mice. The relative expression were calculated based the Gray value of immunoblotting band(c). The data are expressed as

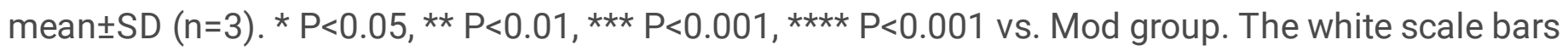
represent $50 \mu \mathrm{m}$. 
a

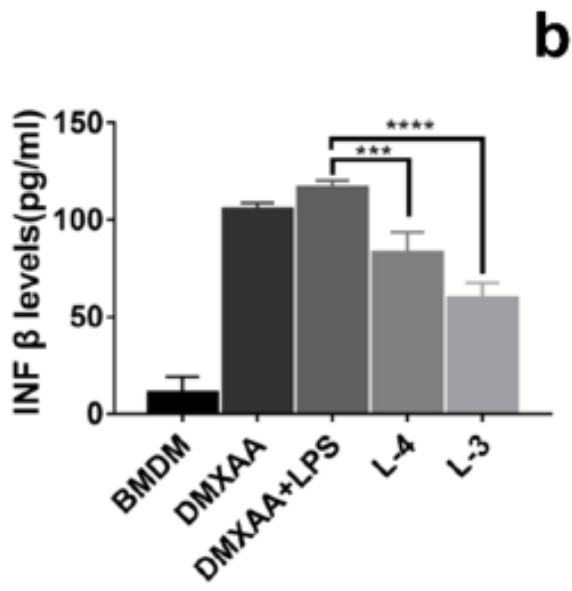

\section{b}

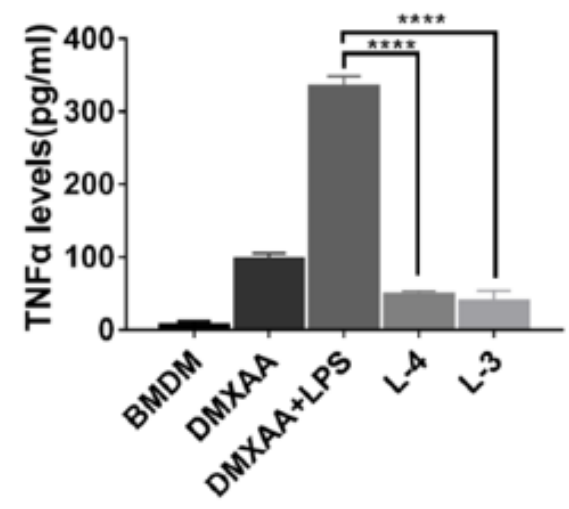

d

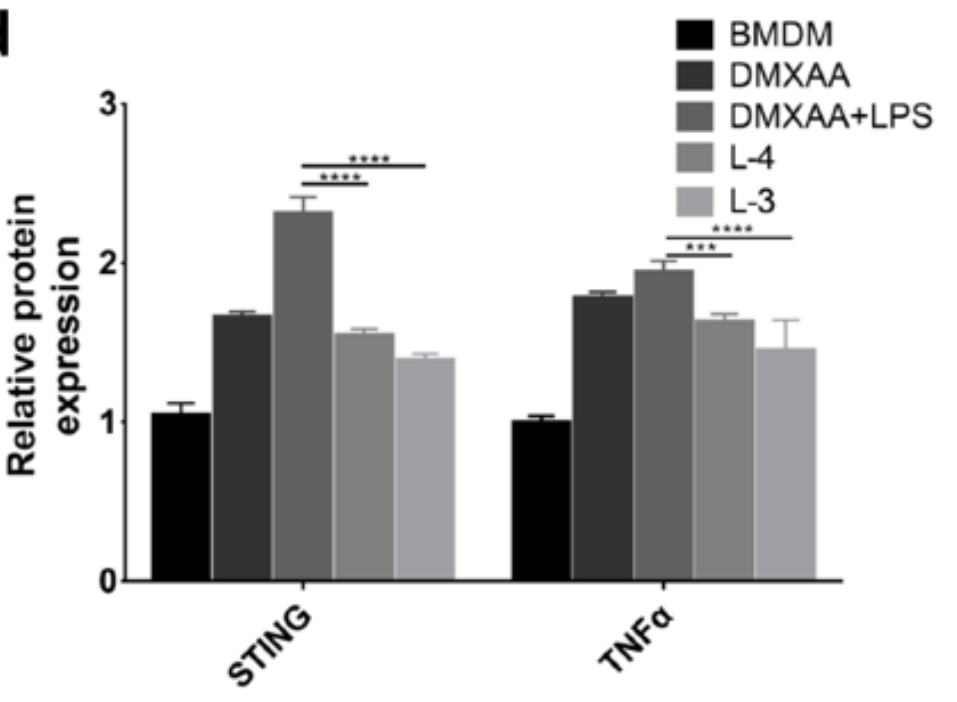

C

DMXAA - ++++ LPS - - + ++ P-TBK1

TBK1

STING

TNFa

Tubulin

LGZG

e

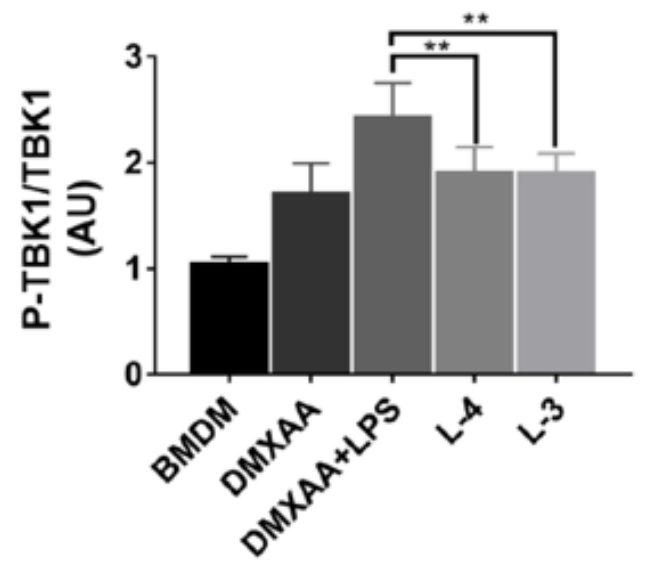

Figure 6

Effects of LGZG on STING-TBK1-NF-KB pathway in BMDMs. a, b Concentration of IFN $\beta(a)$ and TNFa(b) from the culture media of BMDMs treated with DMXAA, LPS and two doses of LGZG. BMDMs were treated with DMXAA $(75 \mathrm{mg} / \mathrm{mL})$ or control $(7.5 \%$ NaHCO3) with or without LGZG (10-4or $10-3 \mathrm{~g} / \mathrm{mL})$ for 24 hours in the absence or presence of LPS $(100 \mathrm{ng} / \mathrm{mL})$. c Immunoblotting analysis of the expression of TBK1, p-TBK1,STING and TNFa. d Quantification of protein levels of STING and TNFa. e Quantification of

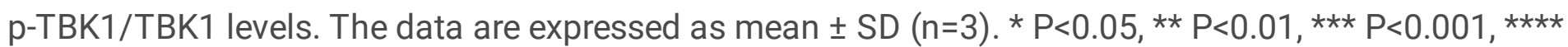
$P<0.001$ vs. DMXAA+LPS. 
a

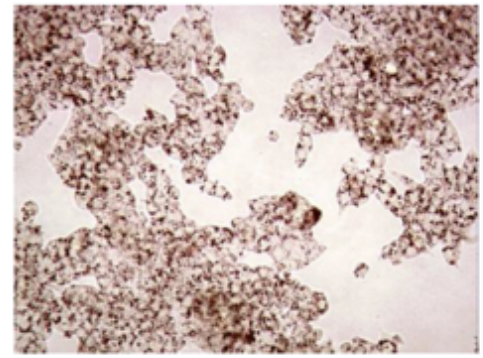

BMDM+HepG2

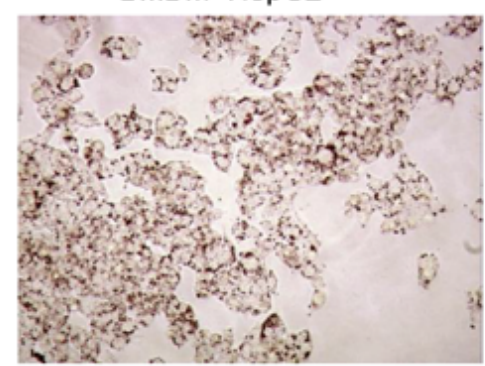

$\mathrm{DMXAA}+\mathrm{BMDM}+\mathrm{HepG} 2+\mathrm{L}^{-4}$

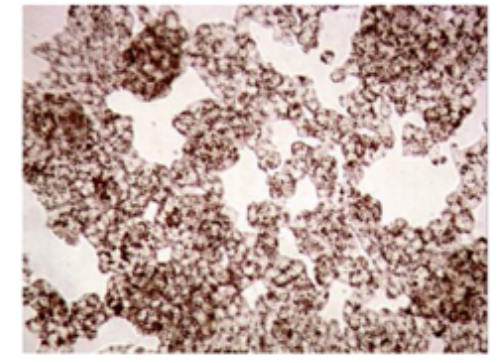

DMXAA+BMDM+HepG2

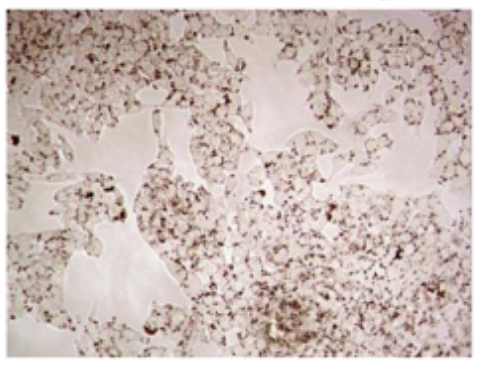

$\mathrm{DMXAA}+\mathrm{BMDM}+\mathrm{HepG} 2+\mathrm{L}^{-3}$ b

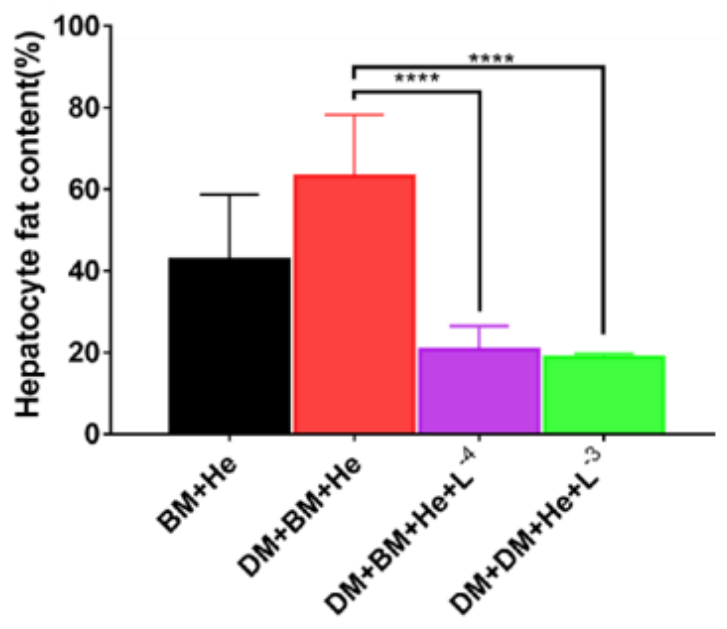

\section{Figure 7}

Macrophages treated with Lingguizhugan Decoction reduced lipid deposition in liver cells. a Hepatocyte fat deposition of co-cultures. HepG2 cells were incubated in the absence of macrophages or co-cultured with BMDMs from WT mice for 48 hours and treated with DMXAA $(75 \mathrm{mg} / \mathrm{mL})$ or control in the presence of PA ( $250 \mathrm{mmol} / \mathrm{L})$ for the last 24 hours. Before harvest, hepatocytes or cocultures were stained with oil red $O$ for 1 hour. $b$ Bar graph displays quantification of fat content. 